\title{
TIME RESOLVED PHASE SPACE TOMOGRAPHY AT FLASH USING A TRANSVERSE DEFLECTING RF-STRUCTURE
}

\author{
Michael Röhrs*, Christopher Gerth, Holger Schlarb \\ Deutsches Elektronen-Synchrotron DESY, D-22603 Hamburg, Germany
}

\begin{abstract}
To initiate Self-Amplification of Spontaneous Emission (SASE) in single-pass Free Electron Lasers (FEL), electron bunches with high peak current and small slice emittance are necessary. At FLASH at DESY, this is accomplished by longitudinal shortening in two magnetic bunch compressors. The compression process may be accompanied by distortions from coherent synchrotron radiation (CSR) and space charge (SC) forces. Their effect on transverse phase space can be studied with a vertically deflecting RF-structure, which allows to measure the horizontal slice emittance and, in combination with tomographic methods, the horizontal phase space distribution within 'time slices'. In this paper, experimental results for SASE operation at $27 \mathrm{~nm}$ are presented. Bunch fractions with a normalized emittance of $3 \mu \mathrm{m}$ and a current of $1 \mathrm{kA}$ have been detected. CSR effects have been found to be the dominating source for slice emittance growth.
\end{abstract}

\section{INTRODUCTION}

The Free electron LASer in Hamburg (FLASH) is a SASE-FEL providing radiation at a fundamental wavelength which can be tuned from $47 \mathrm{~nm}$ down to $13 \mathrm{~nm}$ [1]. The radiation pulses have a duration of 10-50 fs. The bunch fractions producing these pulses are correspondingly short, which makes it a challenging task to experimentally determine properties like current and emittance. The most powerful tools for this purpose are currently traveling wave transverse deflecting RF-structures [2]. A bunch traversing such a structure (here:vertically deflecting) at an appropriate RF phase experiences a vertical kick which depends linearly on time and vanishes in the bunch center. As a consequence, the vertical positions of the bunch electrons downstream of the structure are linearly correlated with their longitudinal coordinates. Standard OTR screens then allow for measurements of the particle distribution in the longitudinal-horizontal plane. Alongside the measurement of the longitudinal charge density profile, this technique permits to determine the horizontal slice emittance by scanning quadrupoles upstream of the structure [3].

\section{EXPERIMENTAL SETUP}

Fig. 1 shows a schematic of the FLASH linac (top). Electron bunches are generated in an RF photocathode (gun) and accelerated in five superconducting modules ACC1 to

\footnotetext{
*michael.roehrs@desy.de
}

ACC5. The bunches are longitudinally shortened in two magnetic bunch compressors BC1 and BC2 at energies of typically $127 \mathrm{MeV}$ and $360 \mathrm{MeV}$, respectively. A dispersive section is used to collimate the beam before it enters the undulator section. The linac was operated with a bunch charge of $0.72 \mathrm{nC}$ and a final energy of $490 \mathrm{MeV}$. We obtained an average radiation energy of $10 \mu \mathrm{J}$ per electron bunch at a fundamental wavelength of $27 \mathrm{~nm}$. The phase of module ACC1 was measured to be $-8.8 \pm 0.5^{\circ}$, which is $3.2^{\circ}$ above the phase for maximum peak current $\left(-12^{\circ}\right)$. The phases of modules ACC23 and ACC45 were set to $-11 \pm 0.5^{\circ}$ and $0 \pm 0.5^{\circ}$, respectively.

An S-band vertically deflecting RF-structure(LOLA) operating at a frequency of $2.856 \mathrm{GHz}$ is installed at the end of the linac (Fig. 1, bottom). $10 \mathrm{~m}$ downstream of LOLA, an OTR screen in combination with a 12 Bit CCD camera is installed to measure the transverse (LOLA off) and longitudinal-horizontal (LOLA on) profiles of single bunches [4]. A horizontal kicker synchronized to the RF is used to steer single bunches onto the screen, which is mounted $10 \mathrm{~mm}$ off-axis. The time intervals corresponding to vertical offsets on the screen can be determined with high accuracy by monitoring the vertical bunch position during a scan of the RF phase in LOLA. The r.m.s. time resolution of measured longitudinal profiles may be estimated by the vertical r.m.s. beam size at the screen location with LOLA switched off. In case of the presented measurement, the conversion constant between vertical offset and time is $10 \pm 0.1 \mathrm{~mm} / \mathrm{ps}$, and the r.m.s. time resolution is $24 \mathrm{fs}$.

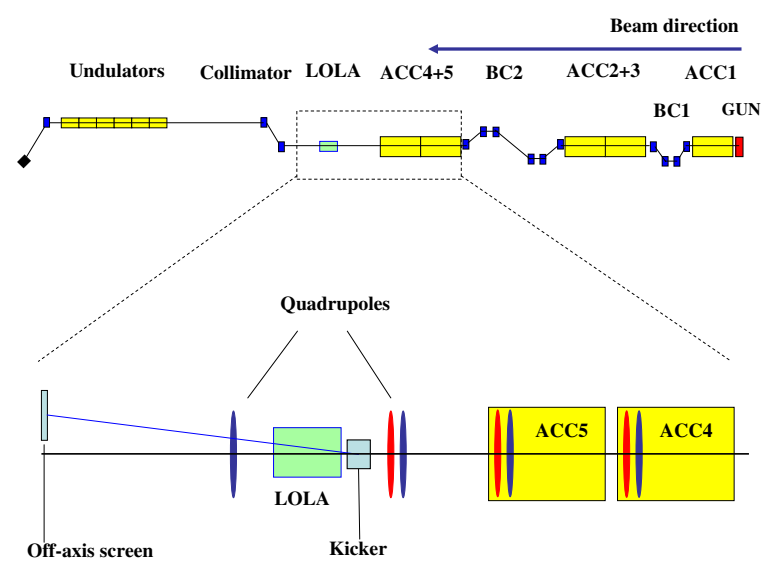

Figure 1: Sketch of the FLASH beam line (top) and the section used for the experiment (bottom). 
The optics for the quadrupole scan has been designed to maintain a constant vertical beam size at the screen during the scan so the time resolution is unchanged. At the same time, the horizontal phase advance between the first changed quadrupole and the screen is changed by $180 \mathrm{de}-$ grees in 14 equidistant steps.

\section{PHASE SPACE RECONSTRUCTION}

Since a quadrupole scan causes basically rotations in transverse phase space, the distributions in horizontal phase space may be reconstructed from measured horizontal density profiles during the scan via tomographic methods. There are several tomography algorithms used in beam physics, which deal differently with the non-uniqueness of the reconstruction resulting from a finite set of experimental data. A particularly well-suited tomography algorithm for nearly gaussian distributions is the so called Maximum Entropy Algorithm [5], which compensates for the missing information by maximizing the entropy of the phase space distribution and thus minimizing substructure. Here, an implementation of the algorithm in $\mathrm{C}++[6,7]$ has been used.

In combination with a transverse deflecting structure, the horizontal phase space distribution of longitudinal sections of the bunch may be reconstructed correspondingly. For this purpose, each recorded OTR image has been subdivided into horizontal slices starting from a distinguished reference point in the longitudinal profile. The vertical slice width has been chosen to be the projected vertical r.m.s. width (LOLA off). For comparison, the r.m.s. slice emittance has also been calculated from the r.m.s. widths of the measured horizontal profiles using a standard least square fit (LSQF) method. Great care has been taken to include the entire detected beam intensity for determining the profiles.

The measurement has been simulated by tracking a particle distribution to the position of the first scanned

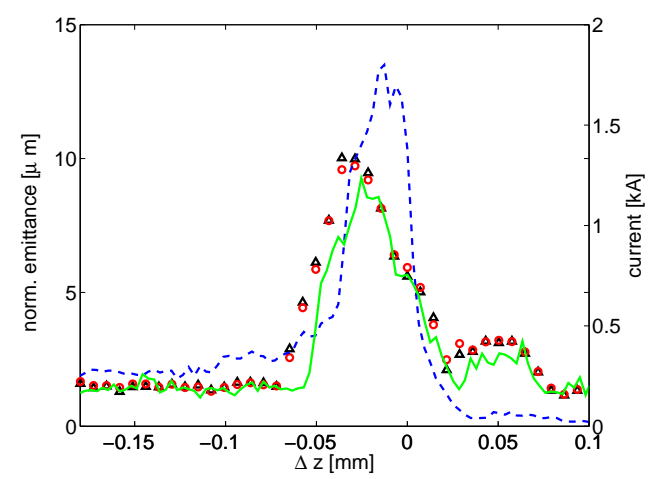

Figure 2: Simulation of the measurement. Reconstructed normalized emittance using a LSQF (red circles) and tomography (black triangles), original slice emittance (green solid line) and current profile (blue dashed line, axis on the right hand side). The front of the bunch is on the right hand side. quadrupole, and then for each quadrupole setting to the position of the screen. From the resulting transverse distributions (including effects from LOLA and the kicker) images have been generated and used as an input for a phase space reconstruction. The particle tracking has been done with ASTRA [8] in straight sections and with CSR-track [9] in the bunch compressors. Fig. 2 shows original slice emittance (green solid line) and current (blue dashed line) of the distribution and the slice emittance obtained via LSQF (red circles) and tomography (black triangles). The simulation shows a well agreement between original and reconstructed emittance except for the rising edge of the current spike. At this position, a limited time resolution and dispersion caused by the kicker lead to deviations. Errors due to chromatic and transverse space charge effects have been found to be negligible. Energy and quadrupole gradient errors may lead to additional deviations of $5 \%$ (r.m.s.).

\section{EXPERIMENTAL RESULTS}

Fig. 3 shows the measured current profile (blue line) and the slice emittance obtained using a LSQF (red circles) and tomography (black triangles). The current spike has a width (FWHM) of $33 \mu \mathrm{m}$ and peaks at $1.6 \mathrm{kA}$. The slice emittance ranges from $2 \mu \mathrm{m}$ in the tail to more than $10 \mu \mathrm{m}$ in the spike. The result of both methods is in good agreement except for the rising edge of the spike, where the tomographic reconstruction produces artefacts due to the limited time resolution. The reconstructed distribution in horizontal phase space for distinguished slices designated in Fig 3 is shown in Fig 4. The phase space distribution in the tail (Fig. 4a) is nearly gaussian-shaped. After applying an intensity threshold cutting $10 \%$ of the charge the emittance reduces to $1.5 \mu \mathrm{m}$. Both values agree well with projected emittance measurements in the injector. At the rising edge of the current spike the emittance increases due to a bifurcation in horizontal phase space (Fig. 4b). At the front

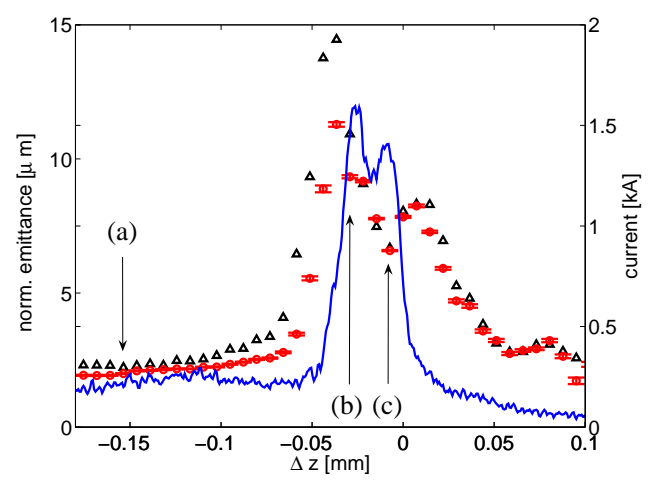

Figure 3: Experimental results. Measured normalized slice emittance using a LSQF (red circles) and tomography (black triangles), current profile (blue dashed line, axis on the right hand side). The arrows designate the longitudinal slice positions corresponding to the phase space distributions shown in Fig. 4. 


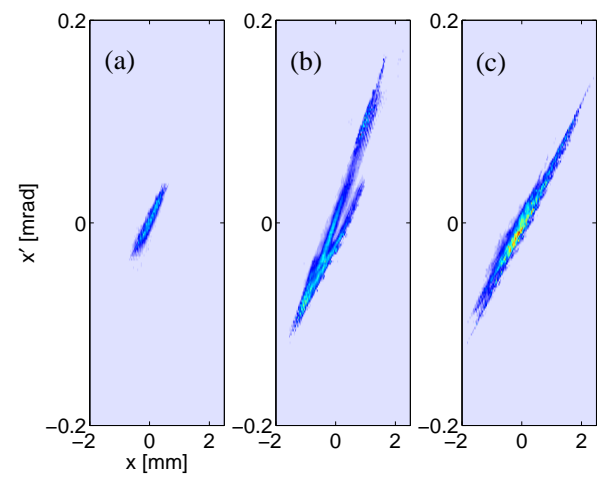

Figure 4: Measured horizontal phase space of time slices designated in Fig. 3.

Table 1: Current and normalized emittance after applying intensity cuts in horizontal phase space. The data refer to the distribution shown in Fig. 4c.

\begin{tabular}{|l|c|c|}
\hline $\begin{array}{l}\text { charge } \\
\text { fraction [\%] }\end{array}$ & current [kA] & emittance $[\mu \mathbf{m}]$ \\
\hline 100 & 1.3 & 6.7 \\
90 & 1.2 & 4.1 \\
80 & 1.0 & 3.3 \\
70 & 0.9 & 2.5 \\
60 & 0.8 & 2.0 \\
\hline
\end{tabular}

of the spike the distortions vanish again and a high density core appears (Fig. 4c). Table 1 shows current and emittance in this slice after applying different intensity thresholds. A cut of $20 \%$ of the particles results in an emittance of about $3 \mu \mathrm{m}$ while the current is still about $1 \mathrm{kA}$. .

\section{COMPARISON WITH SIMULATIONS}

In case of SASE operation, current and emittance of the lasing bunch fraction may be estimated from observed radiation properties by means of a simulation of the FEL process [1]. In this way, a peak current of 2-3 kA and a transverse normalized r.m.s. emittance of 1-1.5 $\mu \mathrm{m}$ have been determined for operation at $13 \mathrm{~nm}$. At larger wave lengths as in the presented measurement, the emittance is expected to be slightly larger, but still below $4 \mu \mathrm{m}$ [10], which would be in agreement with the presented results. However, since final compression is accomplished downstream of LOLA in the collimator, the results in particular for the peak current can unfortunately not be directly compared to these predictions.

Simulations using ASTRA and CSR-track have been performed to investigate if the measured slice emittance may be reproduced. The measurement of the RF phase of the first acceleration module turned out to be a problem here, since the accuracy is not sufficient to determine the resulting bunch properties accurately. The phase has therefore been fine-tuned so the measured width of the cur- rent spike and the peak current are reproduced within $10 \%$. Fig. 5 shows a comparison of the slice emittance obtained in the simulation (black solid line) and the measured slice emittance (red circles). The deviations at the rising edge of the spike may be explained by a limited time resolution and dispersion as already discussed. The deviation at the falling edge cannot be explained satisfyingly up to now. The strong increase in slice emittance in the current spike is largely due to CSR effects BC2, as can be seen from a simulation neglecting CSR forces in BC2 (blue dashed line). This is confirmed by the observed bifurcation in horizontal phase space shown in Fig. $4 b$, which can qualitatively be explained by CSR.

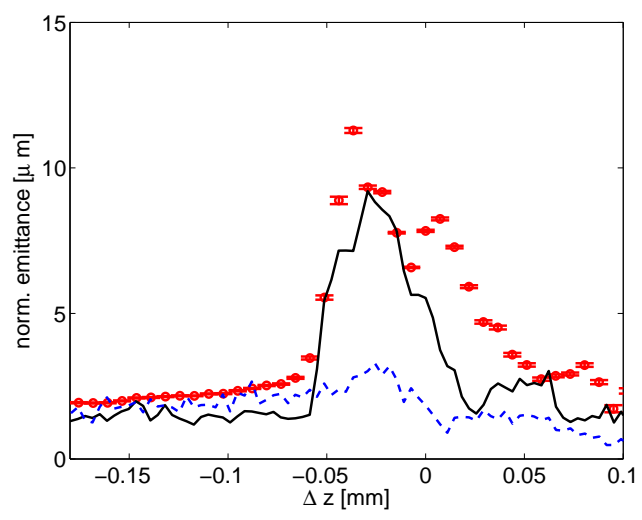

Figure 5: Comparison with simulations. Measured slice emittance using a LSQF (red circles), slice emittance obtained from a simulation including CSR and SC effects (black solid line) and neglecting CSR effects in the second bunch compressor (blue dashed line).

\section{ACKNOWLEDGMENT}

The authors would like to thank the FLASH team, in particular M. Dohlus, I. Zagorodnov, F. Löhl and M. Hüning for their support.

\section{REFERENCES}

[1] W. Ackermann et al., Nature Photonics, vol.1, 2007, p. 336

[2] G. A. Loew et al., SLAC-PUB-135.

[3] P. Emma et al.,LCLS-TN-00-12.

[4] M. Huening et al.,FEL 2005, p. 538.

[5] G. Minerbo, Computer Graphics and Image Processing, 10 (1979), p. 48-68

[6] J.J. Scheins,TESLA report 2004-08

[7] F. Löhl et al., Phys. Rev. ST Accel. Beams 9 (2006) 092802.

[8] K. Flöttmann, http://www.desy.de/ ${ }^{\prime m p y f l o . ~}$

[9] M. Dohlus et al., FEL 2004, p. 18.

[10] M. Yurkov, private communication. 